\title{
Two Conservative Difference Schemes for Rosenau-Kawahara Equation
}

\author{
Jinsong $\mathrm{Hu},{ }^{1,2}$ Youcai $\mathrm{Xu}^{1}{ }^{1}$ Bing $\mathrm{Hu},{ }^{1}$ and Xiaoping Xie ${ }^{1}$ \\ ${ }^{1}$ School of Mathematics, Sichuan University, Chengdu 610064, China \\ ${ }^{2}$ School of Mathematics and Computer Engineering, Xihua University, Chengdu 610039, China \\ Correspondence should be addressed to Youcai Xu; xyc@scu.edu.cn
}

Received 14 September 2013; Revised 22 December 2013; Accepted 7 January 2014; Published 18 March 2014

Academic Editor: Ricardo Weder

Copyright (c) 2014 Jinsong Hu et al. This is an open access article distributed under the Creative Commons Attribution License, which permits unrestricted use, distribution, and reproduction in any medium, provided the original work is properly cited.

Two conservative finite difference schemes for the numerical solution of the initialboundary value problem of Rosenau-Kawahara equation are proposed. The difference schemes simulate two conservative quantities of the problem well. The existence and uniqueness of the difference solution are proved. It is shown that the finite difference schemes are of second-order convergence and unconditionally stable. Numerical experiments verify the theoretical results.

\section{Introduction}

In the study of compact discrete systems, the wave-wave and wave-wall interactions cannot be described by the wellknown KdV equation. To overcome this shortcoming of KdV equation, Rosenau proposed the following so-called Rosenau equation in $[1,2]$ :

$$
u_{t}+u_{x x x x t}+u_{x}+u u_{x}=0,
$$

which is usually used to describe the dense discrete system and simulate the long-chain transmission model through an L-C flow in radio and computer fields. Park proved the existence and uniqueness of solution to (1) in [3]. However, it is difficult to find its analytical solution. Therefore, the numerical study of (1) is very significant and attract many scholars (see, e.g., [4-10]).

As the further consideration of nonlinear wave, Zuo obtained Rosenau-Kawahara equation by adding viscous term $+u_{x x x}$ and $-u_{x x x x x}$ to Rosenau equation (1) and studied the solitary solution and periodic solution of this equation in [10]. In [11], Labidi and Biswas got the integral of RosenauKawahara equation by using He's principle. Then the solitary solution and two invariance of a generalized RosenauKawahara equation are investigated in [12]. To our best knowledge, there is no study on the numerical method of
Rosenau-Kawahara equation. Therefore, we will study the difference approximate solution of Rosenau-Kawahara equation with the initial data and boundary conditions as follows:

$$
\begin{gathered}
u_{t}+u_{x x x x t}+u_{x}+\alpha u u_{x}+u_{x x x}-u_{x x x x x}=0, \\
u(x, 0)=u_{0}(x), \quad x \in\left[x_{L}, x_{R}\right], \\
u\left(x_{L}, t\right)=u\left(x_{R}, t\right)=0, \quad u_{x}\left(x_{L}, t\right)=u_{x}\left(x_{R}, t\right)=0, \\
u_{x x}\left(x_{L}, t\right)=u_{x x}\left(x_{R}, t\right)=0, \quad t \in[0, T],
\end{gathered}
$$

where $\alpha$ is a known constant and $u_{0}(x)$ is a smooth function. When $\alpha=1$, the solitary wave solution of (2) is (see [10])

$$
\begin{aligned}
u(x, t)= & \left(-\frac{35}{12}+\frac{35}{156} \sqrt{205}\right) \\
& \times \operatorname{sech}^{4}\left[\frac{1}{2} \sqrt{-13+\sqrt{205}}\left(x-\frac{1}{13} \sqrt{205} t\right)\right] .
\end{aligned}
$$

The initial boundary value problem (2)-(4) is in accordance with the Cauchy problem of (2) when $-x_{L} \gg 0, x_{R} \gg 0$. Hence, the boundary condition (4) is reasonable. It is easy to 
verify that (2)-(4) satisfy the following conservative quantities (see [12]):

$$
\begin{gathered}
Q(t)=\int_{x_{L}}^{x_{R}} u(x, t) d x=\int_{x_{L}}^{x_{R}} u_{0}(x) d x=Q(0), \\
E(t)=\|u\|_{L_{2}}^{2}+\left\|u_{x x}\right\|_{L_{2}}^{2}=E(0),
\end{gathered}
$$

where $Q(0), E(0)$ are both constants only depending on initial data.

It is well known that a reasonable difference scheme has not only high-accuracy but also can maintain some physical properties of original problem. Lots of numerical experiments show that conservative difference scheme can simulate the conservative law of initial problem well since it could avoid the nonlinear blow-up [13-24]. Moreover, it is suitable to compute for long-time. $\mathrm{Li}$ and $\mathrm{Vu}$-Quoc pointed in [14] that in some areas, the ability to preserve some invariant properties of the original differential equation is a criterion to judge the success of a numerical simulation. Therefore, constructing the conservative difference scheme is a significant job.

The rest of this paper is organized as follows. We respectively propose a two-level nonlinear Crank-Nicolson difference scheme and three-level linear difference scheme for initial boundary value problems (2)-(4) in Sections 2 and 3. We analyze its two discrete conservative laws. The existence and uniqueness of the difference solution are proved. It is shown that the finite difference schemes are of second-order convergence and unconditionally stable. In Section 4, we verify our theoretical analysis by numerical experiments.

\section{Nonlinear Crank-Nicolson Conservative Difference Scheme}

In this section, we propose a two-level nonlinear CrankNicolson difference scheme and give the theoretical analysis.

In the rest of this paper, $C$ denotes a general positive constant which may denote different value in different occurrence.

2.1. Nonlinear Difference Scheme and Its Conservative Law. Let $h$ and $\tau$ be the uniform step size in the spatial and temporal direction, respectively. The interval $\left[x_{L}, x_{R}\right]$ is divided into $J$ equal parts, where $J$ is a fixed positive integer. Denote $h=\left(x_{R}-x_{L}\right) / J, x_{j}=x_{L}+j h(j=-2,-1,0, \ldots, J, J+$ $1, J+2), t_{n}=n \tau(n=0,1,2, \ldots, N, N=[T / \tau])$. Let $u_{j}^{n}$ be the difference approximation of $u(x, t)$ at $\left(x_{j}, t_{n}\right)$, that is, $u_{j}^{n} \approx u\left(x_{j}, t_{n}\right)$. Denote $Z_{h}^{0}=\left\{u=\left(u_{j}\right) \mid u_{-2}=u_{-1}=u_{0}=u_{J}=\right.$ $\left.u_{J+1}=u_{J+2}=0, j=-2,-1,0,1, \ldots, J, J+1, J+2\right\}$. We define the difference operators, inner product, and norms that will be used in this paper as follows:

$$
\begin{aligned}
\left(u_{j}^{n}\right)_{x}=\frac{u_{j+1}^{n}-u_{j}^{n}}{h}, & \left(u_{j}^{n}\right)_{\bar{x}}=\frac{u_{j}^{n}-u_{j-1}^{n}}{h}, \\
\left(u_{j}^{n}\right)_{\widehat{x}}=\frac{u_{j+1}^{n}-u_{j-1}^{n}}{2 h}, & \left(u_{j}^{n}\right)_{t}=\frac{u_{j}^{n+1}-u_{j}^{n}}{\tau},
\end{aligned}
$$

$$
\begin{gathered}
\left(u_{j}^{n}\right)_{\hat{t}}=\frac{u_{j}^{n+1}-u_{j}^{n-1}}{2 \tau}, \quad u_{j}^{n+1 / 2}=\frac{u_{j}^{n+1}+u_{j}^{n}}{2}, \\
\bar{u}_{j}^{n}=\frac{u_{j}^{n+1}+u_{j}^{n-1}}{2}, \\
\left\langle u^{n}, v^{n}\right\rangle=h \sum_{j=1}^{J-1} u_{j}^{n} v_{j}^{n}, \quad\left\|u^{n}\right\|^{2}=\left\langle u^{n}, u^{n}\right\rangle, \\
\left\|u^{n}\right\|_{\infty}=\max _{1 \leq j \leq J-1}\left|u_{j}^{n}\right| .
\end{gathered}
$$

Consider the following finite difference scheme for problems (2)-(4):

$$
\begin{gathered}
\left(u_{j}^{n}\right)_{t}+\left(u_{j}^{n}\right)_{x x \overline{x x} t}+\left(u_{j}^{n+1 / 2}\right)_{\widehat{x}} \\
+\left(u_{j}^{n+1 / 2}\right)_{x \bar{x} \widehat{x}}-\left(u_{j}^{n+1 / 2}\right)_{x x \overline{x x} \widehat{x}} \\
+\frac{1}{3} \alpha\left\{u_{j}^{n+1 / 2}\left(u_{j}^{n+1 / 2}\right)_{\widehat{x}}+\left[\left(u_{j}^{n+1 / 2}\right)^{2}\right]_{\widehat{x}}\right\}=0, \\
(j=1,2, \ldots, J-1, n=1,2, \ldots, N-1), \\
u_{j}^{0}=u_{0}\left(x_{j}\right), \quad(j=0,1,2, \ldots, J), \\
u^{n} \in Z_{h}^{0}, \quad\left(u_{0}^{n}\right)_{\widehat{x}}=\left(u_{J}^{n}\right)_{\widehat{x}}=0, \\
\left(u_{0}^{n}\right)_{x \bar{x}}=\left(u_{J}^{n}\right)_{x \bar{x}}=0, \quad(n=0,1,2, \ldots, N) .
\end{gathered}
$$

The discrete boundary condition (11) is reasonable from the boundary condition (4).

Lemma 1. For any two discrete functions $u, v \in Z_{h}^{0}$, we have

$$
\left\langle u_{x}, v\right\rangle=-\left\langle u, v_{\bar{x}}\right\rangle, \quad\left\langle u_{x \bar{x}}, v\right\rangle=-\left\langle u_{x}, v_{x}\right\rangle
$$

from summation by parts (see [22]). Thus,

$$
\left\langle u_{x}, u\right\rangle=-\left\langle u, u_{\bar{x}}\right\rangle, \quad\left\langle u_{x \bar{x}}, u\right\rangle=-\left\langle u_{x}, u_{x}\right\rangle=-\left\|u_{x}\right\|^{2} .
$$

And if $\left(u_{0}^{n}\right)_{x \bar{x}}=\left(u_{J}^{n}\right)_{x \bar{x}}=0$, then

$$
\left\langle u_{x x \overline{x x}}, u\right\rangle=\left\|u_{x x}\right\|^{2} .
$$

The following theorem shows how the difference schemes (9)-(11) simulate the conservative law numerically.

Theorem 2. Suppose that $u_{0} \in H_{0}^{2}\left[x_{L}, x_{R}\right]$; then the difference schemes (9)-(11) are conservative for discrete energy; that is,

$$
\begin{gathered}
Q^{n}=h \sum_{j=1}^{J-1} u_{j}^{n}=Q^{n-1}=\cdots=Q^{0}, \\
E^{n}=\left\|u^{n}\right\|^{2}+\left\|u_{x x}^{n}\right\|^{2}=E^{n-1}=\cdots=E^{0} .
\end{gathered}
$$

Proof. Multiplying $h$ in the two sides of (9) and summing up for $j$ from 1 to $J-1$, from boundary (11) and Lemma 1, we obtain

$$
h \sum_{j=1}^{J-1} \frac{u_{j}^{n+1}-u_{j}^{n}}{\tau}=0 .
$$

From the definition of $Q^{n}$, (15) is deduced from (17). 
Taking the inner of (9) with $2 u^{n+1 / 2}$ (i.e., $\left.u^{n+1}+u^{n}\right)$, from boundary (11) and Lemma 1, we get

$$
\begin{aligned}
& \left\|u^{n}\right\|_{t}^{2}+\left\|u_{x x}^{n}\right\|_{t}^{2}+2\left\langle u_{\widehat{x}}^{n+1 / 2}, u^{n+1 / 2}\right\rangle \\
& \quad+2\left\langle u_{x \bar{x} \hat{x}}^{n+1 / 2}, u^{n+1 / 2}\right\rangle-2\left\langle u_{x x \bar{x} x \hat{x}}^{n+1 / 2} u^{n+1 / 2}\right\rangle \\
& \quad+2 \alpha\left\langle\varphi\left(u^{n+1 / 2}\right), u^{n+1 / 2}\right\rangle=0
\end{aligned}
$$

where $\varphi\left(u_{j}^{n+1 / 2}\right)=(1 / 3)\left\{u_{j}^{n+1 / 2}\left(u_{j}^{n+1 / 2}\right)_{\widehat{x}}+\left[\left(u_{j}^{n+1 / 2}\right)^{2}\right]_{\widehat{x}}\right\}$. On the other hand,

$$
\begin{gathered}
\left\langle u_{\widehat{x}}^{n+1 / 2}, u^{n+1 / 2}\right\rangle=0 \\
\left\langle u_{x \bar{x} \hat{x}}^{n+1 / 2}, u^{n+1 / 2}\right\rangle=0 \\
\left\langle u_{x x \bar{x} x \hat{x}}^{n+1 / 2}, u^{n+1 / 2}\right\rangle=0 \\
\left\langle\varphi\left(u^{n+1 / 2}\right), u^{n+1 / 2}\right\rangle \\
=\frac{1}{3} h \sum_{j=1}^{J-1}\left\{u_{j}^{n+1 / 2}\left(u_{j}^{n+1 / 2}\right)_{\widehat{x}}+\left[\left(u_{j}^{n+1 / 2}\right)^{2}\right]_{\widehat{x}}\right\} u_{j}^{n+1 / 2} \\
=\frac{1}{3} h \sum_{j=1}^{J-1}\left(u_{j}^{n+1 / 2}\right)^{2}\left(u_{j}^{n+1 / 2}\right)_{\widehat{x}}+\frac{1}{3} h \sum_{j=1}^{J-1}\left[\left(u_{j}^{n+1 / 2}\right)^{2}\right]_{\widehat{x}} u_{j}^{n+1 / 2} \\
=\frac{1}{3} h \sum_{j=1}^{J-1}\left(u_{j}^{n+1 / 2}\right)^{2}\left(u_{j}^{n+1 / 2}\right)_{\widehat{x}}-\frac{1}{3} h \sum_{j=1}^{J-1}\left(u_{j}^{n+1 / 2}\right)^{2} \\
\times\left(u_{j}^{n+1 / 2}\right)_{\widehat{x}}=0 .
\end{gathered}
$$

Substituting (19)-(22) into (18), we get

$$
\left(\left\|u^{n+1}\right\|^{2}-\left\|u^{n}\right\|^{2}\right)+\left(\left\|u_{x x}^{n+1}\right\|^{2}-\left\|u_{x x}^{n}\right\|^{2}\right)=0 .
$$

From the definition of $E^{n}$, we get (16) by deducing (23).

2.2. Solvability of the Difference Scheme. In order to prove the solvability of difference scheme, we present the following Brouwer fixed point theorem [25].

Lemma 3 (Brouwer fixed point theorem). Let $H$ be a finite dimensional inner product space; suppose that $g: H \rightarrow H$ is continuous and there exists an $\alpha>0$ such that $\langle g(x), x\rangle>0$ for all $x \in H$ with $\|x\|=\alpha$. Then there exists $x^{*} \in H$ such that $g\left(x^{*}\right)=0$ and $\left\|x^{*}\right\| \leq \alpha$.

Theorem 4. There exists $u^{n} \in Z_{h}^{0}(1 \leq n \leq N)$ that satisfies difference schemes (9)-(11).

Proof. We use the mathematical induction to prove our theorem.

Suppose that there exist $u^{0}, u^{1}, \ldots, u^{n}$ that satisfy difference scheme (9) for $n \leq N-1$. Next we prove that there exists $u^{n+1}$ that satisfies difference scheme.
Let $g$ be an operator on $Z_{h}^{0}$ defined by

$$
\begin{aligned}
g(v)= & 2 v-2 u^{n}+2 v_{x x \overline{x x}}-2 u_{x x \overline{x x}}^{n} \\
& +\tau v_{\widehat{x}}+\tau v_{x \bar{x} \hat{x}}-\tau v_{x x \overline{x x} \hat{x}}+\tau \alpha \varphi(v) .
\end{aligned}
$$

Taking the inner product of (24) with $v$, and noticing that

$$
\begin{gathered}
\left\langle v_{\widehat{x}}, v\right\rangle=0, \quad\left\langle v_{x \bar{x} \hat{x}}, v\right\rangle=0, \\
\left\langle v_{x x \overline{x x} \hat{x}}, v\right\rangle=0, \quad\langle\varphi(v), v\rangle=0,
\end{gathered}
$$

we have

$$
\begin{aligned}
\langle g(v), v\rangle= & 2\|v\|^{2}-2\left\langle u^{n}, v\right\rangle+2\left\|v_{x x}\right\|^{2}-2\left\langle u_{x x}^{n}, v_{x x}\right\rangle \\
\geq & 2\|v\|^{2}-2\left\|u^{n}\right\| \cdot\|v\|+2\left\|v_{x x}\right\|^{2}-2\left\|u_{x x}^{n}\right\| \cdot\left\|v_{x x}\right\| \\
\geq & 2\|v\|^{2}-\left(\left\|u^{n}\right\|^{2}+\|v\|^{2}\right)+2\left\|v_{x x}\right\|^{2} \\
& -\left(\left\|u_{x x}^{n}\right\|^{2}+\left\|v_{x x}\right\|^{2}\right) \\
\geq & \|v\|^{2}-\left(\left\|u^{n}\right\|^{2}+\left\|u_{x x}^{n}\right\|^{2}\right)+\left\|v_{x x}^{n}\right\|^{2} \\
\geq & \|v\|^{2}-\left(\left\|u^{n}\right\|^{2}+\left\|u_{x x}^{n}\right\|^{2}\right) .
\end{aligned}
$$

Therefore, for any $v \in Z_{h}^{0}$, if $\|v\|^{2}=\left\|u^{n}\right\|^{2}+\left\|u_{x x}^{n}\right\|^{2}+1$, then $\langle g(v), v\rangle>0$. From Lemma 3, there exists $v^{*} \in Z_{h}^{0}$ such that $g\left(v^{*}\right)=0$. Choose $u^{n+1}=2 v^{*}-u^{n}$. It is easy to verify that $u^{n+1}$ satisfies difference scheme (9).

2.3. Convergence, Stability, and Uniqueness of Solution. Suppose that $v(x, t)$ is the solution to (2)-(4). Denote $v_{j}^{n}=$ $v\left(x_{j}, t_{n}\right)$. Then the truncation error of difference schemes (9)(11) is

$$
\begin{aligned}
r_{j}^{n}=\left(v_{j}^{n}\right)_{t}+\left(v_{j}^{n}\right)_{x x \bar{x} \bar{x} t}+\left(v_{j}^{n+1 / 2}\right)_{\widehat{x}} \\
+\left(v_{j}^{n+1 / 2}\right)_{x \bar{x} \widehat{x}}-\left(v_{j}^{n+1 / 2}\right)_{x x \bar{x} \bar{x}}+\alpha \varphi\left(v_{j}^{n+1 / 2}\right), \\
(j=1,2, \ldots, J-1, n=1,2, \ldots, N-1), \\
v_{j}^{0}=u_{0}\left(x_{j}\right), \quad(j=0,1,2, \ldots, J), \\
v^{n} \in Z_{h}^{0}, \quad\left(v_{0}^{n}\right)_{\widehat{x}}=\left(v_{J}^{n}\right)_{\widehat{x}}=0, \\
\left(v_{0}^{n}\right)_{x \bar{x}}=\left(v_{J}^{n}\right)_{x \bar{x}}=0, \quad(n=0,1,2, \ldots, N) .
\end{aligned}
$$

By Taylor expansion, we know that $r_{j}^{n}=O\left(\tau^{2}+h^{2}\right)$ as $h, \tau \rightarrow$ 0 .

Lemma 5. Suppose that $u_{0} \in H_{0}^{2}\left[x_{L}, x_{R}\right]$. Then the solution of problems (2)-(4) satisfies

$$
\begin{gathered}
\|u\|_{L_{2}} \leq C, \quad\left\|u_{x}\right\|_{L_{2}} \leq C, \quad\left\|u_{x x}\right\|_{L_{2}} \leq C, \\
\|u\|_{L_{\infty}} \leq C, \quad\left\|u_{x}\right\|_{L_{\infty}} \leq C .
\end{gathered}
$$


Proof. It follows from (7) that

$$
\|u\|_{L_{2}} \leq C, \quad\left\|u_{x x}\right\|_{L_{2}} \leq C .
$$

Using Hölder inequality and Cauchy-Schwarz inequality, we can get

$$
\begin{aligned}
\|u\|_{L_{2}}^{2} & =\int_{x_{L}}^{x_{R}} u_{x} u_{x} d x=\left.u u_{x}\right|_{x_{L}} ^{x_{R}}-\int_{x_{L}}^{x_{R}} u u_{x x} d x \\
& =-\int_{x_{L}}^{x_{R}} u u_{x x} d x \leq\|u\|_{L_{2}} \cdot\left\|u_{x x}\right\|_{L_{2}} \\
& \leq \frac{1}{2}\left(\|u\|_{L_{2}}^{2}+\left\|u_{x x}\right\|_{L_{2}}^{2}\right) .
\end{aligned}
$$

Thus,

$$
\left\|u_{x}\right\|_{L_{2}} \leq C
$$

which also yields $\|u\|_{L_{\infty}} \leq C,\left\|u_{x}\right\|_{L_{\infty}} \leq C$ from Sobolev inequality.

Lemma 6 (discrete Sobolev inequality [22]). There exist constants $C_{1}$ and $C_{2}$ such that

$$
\left\|u^{n}\right\|_{\infty} \leq C_{1}\left\|u^{n}\right\|+C_{2}\left\|u_{x}^{n}\right\| .
$$

Lemma 7 (discrete Gronwall inequality [22]). Assume that $w(k), \rho(k)$ are nonnegative gridding functions, and $\rho(k)$ is increasing. If $C>0$, and for any $k$

$$
w(k) \leq \rho(k)+C \tau \sum_{l=0}^{k-1} w(l),
$$

then for any $k$

$$
w(k) \leq \rho(k) e^{C \tau k}
$$

Theorem 8. Suppose that $u_{0} \in H_{0}^{2}\left[x_{L}, x_{R}\right]$. Then the solution of difference schemes (9)-(11) satisfies $\left\|u^{n}\right\| \leq C,\left\|u_{x}^{n}\right\| \leq C$, $\left\|u_{x x}^{n}\right\| \leq C$ thus $\left\|u^{n}\right\|_{\infty} \leq C,\left\|u_{x}^{n}\right\|_{\infty} \leq C(n=1,2, \ldots, N)$.

Proof. From (16), we have

$$
\left\|u^{n}\right\| \leq C, \quad\left\|u_{x x}^{n}\right\| \leq C .
$$

From (13) and by Cauchy-Schwarz inequality

$$
\left\|u_{x}^{n}\right\|^{2} \leq\left\|u^{n}\right\| \cdot\left\|u_{x x}^{n}\right\| \leq \frac{1}{2}\left(\left\|u^{n}\right\|^{2}+\left\|u_{x x}^{n}\right\|^{2}\right) \leq C .
$$

Thus,

$$
\left\|u_{x}^{n}\right\| \leq C
$$

Therefore, from Lemma 6, we obtain $\left\|u^{n}\right\|_{\infty} \leq C,\left\|u_{x}^{n}\right\|_{\infty} \leq$ C.

Remark 9. Theorem 8 implies that the solution of difference schemes (9)-(11) is unconditionally stable in the sense of norm $\|\cdot\|_{\infty}$.
Theorem 10. Suppose that $u_{0} \in H_{0}^{2}\left[x_{L}, x_{R}\right]$. Then the solution of difference schemes (9)-(11) $u^{n}$ converges to the solution of (2)-(4) in the sense of norm $\|\cdot\|_{\infty}$, and the convergent rate is $O\left(\tau^{2}+h^{2}\right)$.

Proof. Subtracting (9)-(11) from (27)-(29) and denoting $e_{j}^{n}=$ $v_{j}^{n}-u_{j}^{n}$, we have

$$
\begin{array}{r}
r_{j}^{n}=\left(e_{j}^{n}\right)_{t}+\left(e_{j}^{n}\right)_{x x \bar{x} \bar{x} t}+\left(e_{j}^{n+1 / 2}\right)_{\widehat{x}}+\left(e_{j}^{n+1 / 2}\right)_{x \bar{x} \hat{x}} \\
-\left(e_{j}^{n+1 / 2}\right)_{x x \overline{x x} \hat{x}}+\alpha \varphi\left(v_{j}^{n+1 / 2}\right)-\alpha \varphi\left(u_{j}^{n+1 / 2}\right), \\
(j=1,2, \ldots, J-1, n=1,2, \ldots, N-1), \\
e_{j}^{0}=0, \quad(j=0,1,2, \ldots, J), \\
e^{n} \in Z_{h}^{0}, \quad\left(e_{0}^{n}\right)_{\widehat{x}}=\left(e_{J}^{n}\right)_{\widehat{x}}=0, \\
\left(e_{0}^{n}\right)_{x \bar{x}}=\left(e_{J}^{n}\right)_{x \bar{x}}=0, \quad(n=0,1,2, \ldots, N) .
\end{array}
$$

Taking the inner product of (40) with $2 e^{n+1 / 2}$, we get

$$
\begin{aligned}
\left\langle r^{n}, 2 e^{n+1 / 2}\right\rangle= & \left\|e^{n}\right\|_{t}^{2}+\left\|e_{x x}^{n}\right\|_{t}^{2}+\left\langle e_{\widehat{x}}^{n+1 / 2}, 2 e^{n+1 / 2}\right\rangle \\
& +\left\langle e_{x \bar{x} \hat{x}}^{n+1 / 2}, 2 e^{n+1 / 2}\right\rangle-\left\langle e_{x x \bar{x} x \widehat{x}}^{n+1 / 2}, 2 e^{n+1 / 2}\right\rangle \\
& +\alpha\left\langle\varphi\left(v^{n+1 / 2}\right)-\varphi\left(u^{n+1 / 2}\right), 2 e^{n+1 / 2}\right\rangle .
\end{aligned}
$$

Similar to (19)-(21), we have

$$
\begin{aligned}
& \left\langle e_{\widehat{x}}^{n+1 / 2}, 2 e^{n+1 / 2}\right\rangle=0, \\
& \left\langle e_{x \bar{x} \hat{x}}^{n+1 / 2}, 2 e^{n+1 / 2}\right\rangle=0, \\
& \left\langle e_{x x \bar{x} \bar{x} \widehat{x}}^{n+1 / 2}, 2 e^{n+1 / 2}\right\rangle=0 .
\end{aligned}
$$

So, (43) can be rewritten as

$$
\begin{aligned}
& \left(\left\|e^{n+1}\right\|^{2}-\left\|e^{n}\right\|^{2}\right)+\left(\left\|e_{x x}^{n+1}\right\|^{2}-\left\|e_{x x}^{n}\right\|^{2}\right) \\
& =\tau\left\langle r^{n}, 2 e^{n+1 / 2}\right\rangle-\alpha \tau\left\langle\varphi\left(v^{n+1 / 2}\right)-\varphi\left(u^{n+1 / 2}\right), 2 e^{n+1 / 2}\right\rangle .
\end{aligned}
$$

As

$$
\begin{aligned}
-\langle & \left.\left(v^{n+1 / 2}\right)-\varphi\left(u^{n+1 / 2}\right), 2 e^{n+1 / 2}\right\rangle \\
= & \frac{-2 h}{3} \sum_{j=1}^{J-1}\left[v_{j}^{n+1 / 2}\left(v_{j}^{n+1 / 2}\right)_{\widehat{x}}-u_{j}^{n+1 / 2}\left(u_{j}^{n+1 / 2}\right)_{\widehat{x}}\right] e_{j}^{n+1 / 2} \\
& -\frac{2 h}{3} \sum_{j=1}^{J-1}\left[\left(v_{j}^{n+1 / 2}\right)^{2}-\left(u_{j}^{n+1 / 2}\right)^{2}\right]_{\widehat{x}} e_{j}^{n+1 / 2} \\
= & \frac{-2 h}{3} \sum_{j=1}^{J-1}\left[v_{j}^{n+1 / 2}\left(e_{j}^{n+1 / 2}\right)_{\widehat{x}}+e_{j}^{n+1 / 2}\left(u_{j}^{n+1 / 2}\right)_{\widehat{x}}\right] e_{j}^{n+1 / 2} \\
& +\frac{2 h}{3} \sum_{j=1}^{J-1}\left[e_{j}^{n+1 / 2}\left(v_{j}^{n+1 / 2}+u_{j}^{n+1 / 2}\right)\right]\left(e_{j}^{n+1 / 2}\right)_{\widehat{x}}
\end{aligned}
$$


from Lemma 5 and Theorem 8, we obtain

$$
\begin{gathered}
\left|v_{j}^{n+1 / 2}\right| \leq C, \quad\left|u_{j}^{n+1 / 2}\right| \leq C, \\
\left|\left(u_{j}^{n+1 / 2}\right)_{\widehat{x}}\right| \leq C, \quad(j=0,1,2, \ldots, J) .
\end{gathered}
$$

Using Cauchy-Schwarz inequality, we have

$$
\begin{aligned}
-\alpha & \left\langle\varphi\left(v^{n+1 / 2}\right)-\varphi\left(u^{n+1 / 2}\right), 2 e^{n+1 / 2}\right\rangle \\
\leq & \frac{2}{3} C|\alpha| h \sum_{j=1}^{J-1}\left(\left|\left(e_{j}^{n+1 / 2}\right)_{\widehat{x}}\right|+\left|e_{j}^{n+1 / 2}\right|\right)\left|e_{j}^{n+1 / 2}\right| \\
& +\frac{2}{3} C|\alpha| h \sum_{j=1}^{J-1}\left|e_{j}^{n+1 / 2}\right| \cdot\left|\left(e_{j}^{n+1 / 2}\right)_{\widehat{x}}\right| \\
\leq & C\left(\left\|e^{n+1 / 2}\right\|^{2}+\left\|e_{x}^{n+1 / 2}\right\|^{2}\right) \\
\leq & C\left(\left\|e^{n+1}\right\|^{2}+\left\|e^{n}\right\|^{2}+\left\|e_{x}^{n+1}\right\|^{2}+\left\|e_{x}^{n}\right\|^{2}\right) .
\end{aligned}
$$

On the other hand,

$$
\begin{aligned}
\left\langle r^{n}, 2 e^{n+1 / 2}\right\rangle & =\left\langle r^{n}, e^{n+1}+e^{n}\right\rangle \\
& \leq\left\|r^{n}\right\|^{2}+\frac{1}{2}\left(\left\|e^{n+1}\right\|^{2}+\left\|e^{n}\right\|^{2}\right) .
\end{aligned}
$$

Substituting (48) and (49) into (45), we get

$$
\begin{aligned}
& \left(\left\|e^{n+1}\right\|^{2}-\left\|e^{n}\right\|^{2}\right)+\left(\left\|e_{x x}^{n+1}\right\|^{2}-\left\|e_{x x}^{n}\right\|^{2}\right) \\
& \leq C \tau\left(\left\|e^{n+1}\right\|^{2}+\left\|e^{n}\right\|^{2}+\left\|e_{x}^{n+1}\right\|^{2}+\left\|e_{x}^{n}\right\|^{2}\right)+\tau\left\|r^{n}\right\|^{2} .
\end{aligned}
$$

Similar to the deduction process of (38), one can get

$$
\begin{aligned}
\left\|e_{x}^{n+1}\right\|^{2} & \leq \frac{1}{2}\left(\left\|e^{n+1}\right\|^{2}+\left\|e_{x x}^{n+1}\right\|^{2}\right), \\
\left\|e_{x}^{n}\right\|^{2} & \leq \frac{1}{2}\left(\left\|e^{n}\right\|^{2}+\left\|e_{x x}^{n}\right\|^{2}\right) .
\end{aligned}
$$

Therefore, (50) is changed into

$$
\begin{aligned}
& \left(\left\|e^{n+1}\right\|^{2}-\left\|e^{n}\right\|^{2}\right)+\left(\left\|e_{x x}^{n+1}\right\|^{2}-\left\|e_{x x}^{n}\right\|^{2}\right) \\
& \leq C \tau\left[\left\|e^{n+1}\right\|^{2}+\left\|e^{n}\right\|^{2}+\left\|e_{x x}^{n+1}\right\|^{2}+\left\|e_{x x}^{n}\right\|^{2}\right]+\tau\left\|r^{n}\right\|^{2} .
\end{aligned}
$$

Denoting $B^{n}=\left\|e^{n}\right\|^{2}+\left\|e_{x x}^{n}\right\|^{2},(52)$ is equivalent to

$$
B^{n+1}-B^{n} \leq C \tau\left(B^{n+1}+B^{n}\right)+\tau\left\|r^{n}\right\|^{2},
$$

that is,

$$
(1-C \tau)\left(B^{n+1}-B^{n}\right) \leq 2 C \tau B^{n}+\tau\left\|r^{n}\right\|^{2} .
$$

Choosing sufficiently small $\tau$ such that $1-C \tau>0$, we get

$$
B^{n+1}-B^{n} \leq C \tau B^{n}+C \tau\left\|r^{n}\right\|^{2} .
$$

Summing up (55) from 0 to $n-1$, we get

$$
B^{n} \leq B^{0}+C \tau \sum_{l=0}^{n-1}\left\|r^{l}\right\|^{2}+C \tau \sum_{l=0}^{n-1} B^{l} .
$$

Noticing that

$$
\tau \sum_{l=0}^{n-1}\left\|r^{l}\right\|^{2} \leq n \tau \max _{0 \leq l \leq n-1}\left\|r^{l}\right\|^{2} \leq T \cdot O\left(\tau^{2}+h^{2}\right)^{2},
$$

and $B^{0}=O\left(\tau^{2}+h^{2}\right)^{2}$. we have

$$
B^{n} \leq O\left(\tau^{2}+h^{2}\right)^{2}+C \tau \sum_{l=0}^{n-1} B^{l}
$$

From Lemma 7, we get

$$
B^{n} \leq O\left(\tau^{2}+h^{2}\right)^{2}
$$

that is,

$$
\left\|e^{n}\right\| \leq O\left(\tau^{2}+h^{2}\right), \quad\left\|e_{x x}^{n}\right\| \leq O\left(\tau^{2}+h^{2}\right) .
$$

Noticing equality (51), one can obtain

$$
\left\|e_{x}^{n}\right\| \leq O\left(\tau^{2}+h^{2}\right) .
$$

From Lemma 6, we have

$$
\left\|e^{n}\right\|_{\infty} \leq O\left(\tau^{2}+h^{2}\right) .
$$

Similar to Theorem 10, we have the following theorems.

Theorem 11. The solution to difference schemes (9)-(11) is unique.

\section{A Linear Conservative Difference Scheme}

In this section, we propose a three-level linear conservative difference scheme for (2)-(4) and give the theoretical analysis.

3.1. Linear Difference Scheme and Its Conservative Law. Consider the following finite difference scheme for problems (2)-(4):

$$
\begin{array}{r}
\left(u_{j}^{n}\right)_{\hat{t}}+\left(u_{j}^{n}\right)_{x x \overline{x x} \hat{t}}+\left(\bar{u}_{j}^{n}\right)_{\widehat{x}}+\left(\bar{u}_{j}^{n}\right)_{x \bar{x} \hat{x}} \\
-\left(\bar{u}_{j}^{n}\right)_{x x \bar{x} \bar{x} \hat{x}}+\frac{1}{3} \alpha\left[u_{j}^{n}\left(\bar{u}_{j}^{n}\right)_{\widehat{x}}+\left(u_{j}^{n} \bar{u}_{j}^{n}\right)_{\widehat{x}}\right]=0, \\
(j=1,2, \ldots, J-1, n=1,2, \ldots, N-1), \\
u_{j}^{0}=u_{0}\left(x_{j}\right), \quad(j=0,1,2, \ldots, J), \\
u^{n} \in Z_{h}^{0}, \quad\left(u_{0}^{n}\right)_{\widehat{x}}=\left(u_{J}^{n}\right)_{\widehat{x}}=0, \\
\left(u_{0}^{n}\right)_{x \bar{x}}=\left(u_{J}^{n}\right)_{x \bar{x}}=0, \quad(n=0,1,2, \ldots, N) .
\end{array}
$$


The discrete boundary condition (65) is reasonable from the boundary condition (4).

The following theorem shows how the difference schemes (63)-(65) simulate the conservative law as follows.

Theorem 12. Suppose that $u_{0} \in H_{0}^{2}\left[x_{L}, x_{R}\right]$; then the difference schemes (63)-(65) are conservative for discrete energy; that is,

$$
\begin{aligned}
Q^{n} & =\frac{h}{2} \sum_{j=1}^{J-1}\left(u_{j}^{n+1}+u_{j}^{n}\right)+\frac{h}{6} \tau \sum_{j=1}^{J-1} u_{j}^{n}\left(u_{j}^{n+1}\right)_{\widehat{x}} \\
& =Q^{n-1}=\cdots=Q^{0}, \\
E^{n} & =\frac{1}{2}\left(\left\|u^{n+1}\right\|^{2}+\left\|u^{n}\right\|^{2}\right)+\frac{1}{2}\left(\left\|u_{x x}^{n+1}\right\|^{2}+\left\|u_{x x}^{n}\right\|^{2}\right) \\
& =E^{n-1}=\cdots=E^{0} .
\end{aligned}
$$

Proof. Multiplying $h$ in both sides of (63) and summing up for $j$, from boundary (65) and Lemma 1, we obtain

$$
h \sum_{j=1}^{J-1} \frac{u_{j}^{n+1}-u_{j}^{n-1}}{2 \tau}+\frac{1}{3} h \sum_{j=1}^{J-1} u_{j}^{n}\left(\bar{u}_{j}^{n}\right)_{\widehat{x}}=0 .
$$

Notice that

$$
\begin{aligned}
h \sum_{j=1}^{J-1} u_{j}^{n}\left(\bar{u}_{j}^{n}\right)_{\widehat{x}} & =\frac{h}{2} \sum_{j=1}^{J-1} u_{j}^{n}\left(u_{j}^{n+1}\right)_{\widehat{x}}+\frac{h}{2} \sum_{j=1}^{J-1} u_{j}^{n}\left(u_{j}^{n-1}\right)_{\widehat{x}} \\
& =\frac{h}{2} \sum_{j=1}^{J-1} u_{j}^{n}\left(u_{j}^{n+1}\right)_{\widehat{x}}-\frac{h}{2} \sum_{j=1}^{J-1} u_{j}^{n-1}\left(u_{j}^{n}\right)_{\widehat{x}}
\end{aligned}
$$
(69).

From the definition of $Q^{n},(66)$ is deduced from (68) and

Taking the inner product of (63) with $2 \bar{u}^{n}$, from boundary (65) and Lemma 1, we obtain

$$
\begin{aligned}
& \left\|u^{n}\right\|_{\hat{t}}^{2}+\left\|u_{x x}^{n}\right\|_{\hat{t}}^{2}+2\left\langle\bar{u}_{\widehat{x}}^{n}, \bar{u}^{n}\right\rangle+2\left\langle\bar{u}_{x \bar{x} \hat{x}}^{n}, \bar{u}^{n}\right\rangle \\
& -2\left\langle\bar{u}_{x x \overline{x x} \hat{x}}^{n}, \bar{u}^{n}\right\rangle+2 \alpha\left\langle\psi\left(u^{n}, \bar{u}^{n}\right), u^{n}\right\rangle=0,
\end{aligned}
$$

where $\psi\left(u_{j}^{n}, \bar{u}_{j}^{n}\right)=(1 / 3)\left[u_{j}^{n}\left(\bar{u}_{j}^{n}\right)_{\widehat{x}}+\left(u_{j}^{n} \bar{u}_{j}^{n}\right)_{\widehat{x}}\right]$. On the other hand,

$$
\begin{gathered}
\left\langle\bar{u}_{\widehat{x}}^{n}, \bar{u}^{n}\right\rangle=0, \\
\left\langle\bar{u}_{x \bar{x} \widehat{x}}^{n}, \bar{u}^{n}\right\rangle=0, \\
\left\langle\bar{u}_{x x \bar{x} \bar{x}}^{n}, \bar{u}^{n}\right\rangle=0, \\
\left\langle\psi\left(u^{n}, \bar{u}^{n}\right), \bar{u}^{n}\right\rangle \\
=\frac{1}{3} h \sum_{j=1}^{J-1}\left[u_{j}^{n}\left(\bar{u}_{j}^{n}\right)_{\widehat{x}}+\left(u_{j}^{n} \bar{u}_{j}^{n}\right)_{\widehat{x}}\right] \bar{u}_{j}^{n} \\
=\frac{1}{3} h \sum_{j=1}^{J-1} u_{j}^{n} \bar{u}_{j}^{n}\left(\bar{u}_{j}^{n}\right)_{\widehat{x}}+\frac{1}{3} h \sum_{j=1}^{J-1}\left(u_{j}^{n} \bar{u}_{j}^{n}\right)_{\widehat{x}} \bar{u}_{j}^{n} \\
=\frac{1}{3} h \sum_{j=1}^{J-1} u_{j}^{n} \bar{u}_{j}^{n}\left(\bar{u}_{j}^{n}\right)_{\widehat{x}}-\frac{1}{3} h \sum_{j=1}^{J-1} u_{j}^{n} \bar{u}_{j}^{n}\left(\bar{u}_{j}^{n}\right)_{\widehat{x}}=0 .
\end{gathered}
$$

Substituting (71)-(74) into (70), we obtain

$$
\left(\left\|u^{n+1}\right\|^{2}-\left\|u^{n-1}\right\|^{2}\right)+\left(\left\|u_{x x}^{n+1}\right\|^{2}-\left\|u_{x x}^{n-1}\right\|^{2}\right)=0 .
$$

From the definition of $E^{n}$, we get (67) by deducing (75).

\subsection{Solvability of the Difference Scheme}

Theorem 13. There exists unique solution for difference schemes (63)-(65).

Proof. Use the mathematical induction to prove it. It is obvious that $u^{0}$ is uniquely determined by the initial condition (64). We also can get $u^{1}$ by (9)-(11). Now suppose $u^{0}, u^{1}, \ldots, u^{n}(n \leq N-1)$ is solved uniquely. Consider the equation of (63) for $u^{n+1}$

$$
\begin{gathered}
\frac{1}{2 \tau} u_{j}^{n+1}+\frac{1}{2 \tau}\left(u_{j}^{n+1}\right)_{x x \overline{x x}}+\frac{1}{2}\left(u_{j}^{n+1}\right)_{\widehat{x}} \\
+\frac{1}{2}\left(u_{j}^{n+1}\right)_{x \bar{x} \hat{x}}-\frac{1}{2}\left(u_{j}^{n+1}\right)_{x x \overline{x x} \hat{x}} \\
+\frac{1}{2} \alpha \psi\left(u_{j}^{n}, u_{j}^{n+1}\right)=0 .
\end{gathered}
$$

Taking the inner product of (76) with $u^{n+1}$, from boundary condition (65) and Lemma 1, we get

$$
\begin{gathered}
\frac{1}{2 \tau}\left\|u^{n+1}\right\|^{2}+\left\|u_{x x}^{n+1}\right\|^{2}+\frac{1}{2}\left\langle u_{\widehat{x}}^{n+1}, u^{n+1}\right\rangle+\frac{1}{2}\left\langle u_{x \bar{x} \hat{x}}^{n+1}, u^{n+1}\right\rangle \\
-\frac{1}{2}\left\langle u_{x x \bar{x} x \bar{x}}^{n+1}, u^{n+1}\right\rangle+\frac{1}{2} \alpha\left\langle\psi\left(u^{n}, u^{n+1}\right), u^{n+1}\right\rangle=0 .
\end{gathered}
$$

Similar to (71)-(73), we have

$$
\begin{gathered}
\left\langle u_{\widehat{x}}^{n+1}, u^{n+1}\right\rangle=0, \\
\left\langle u_{x \bar{x} \widehat{x}}^{n+1}, u^{n+1}\right\rangle=0, \\
\left\langle u_{x x \bar{x} x \hat{x}}^{n+1}, u^{n+1}\right\rangle=0 .
\end{gathered}
$$

Notice that

$$
\begin{aligned}
& \frac{1}{2}\left\langle\psi\left(u^{n}, u^{n+1}\right), u^{n+1}\right\rangle \\
& =\frac{1}{6} h \sum_{j=1}^{J-1}\left[u_{j}^{n}\left(u_{j}^{n+1}\right)_{\widehat{x}}+\left(u_{j}^{n} u_{j}^{n+1}\right)_{\widehat{x}}\right] u_{j}^{n+1} \\
& =\frac{1}{6} h \sum_{j=1}^{J-1} u_{j}^{n} u_{j}^{n+1}\left(u_{j}^{n+1}\right)_{\widehat{x}}+\frac{1}{6} h \sum_{j=1}^{J-1}\left(u_{j}^{n} u_{j}^{n+1}\right)_{\widehat{x}} u_{j}^{n+1} \\
& =\frac{1}{6} h \sum_{j=1}^{J-1} u_{j}^{n} u_{j}^{n+1}\left(u_{j}^{n+1}\right)_{\widehat{x}}-\frac{1}{6} h \sum_{j=1}^{J-1} u_{j}^{n} u_{j}^{n+1}\left(u_{j}^{n+1}\right)_{\widehat{x}}=0 .
\end{aligned}
$$

Substituting (78)-(81) into (77), we get

$$
\left\|u^{n+1}\right\|^{2}+\left\|u_{x x}^{n+1}\right\|^{2}=0
$$

that is, (76) only admits zero solution. Therefore, there exists a unique $u_{j}^{n+1}$ that satisfies (63). 
3.3. Convergence and Stability of the Difference Scheme. Suppose that $v(x, t)$ is the solution to (2)-(4). Denote $v_{j}^{n}=$ $v\left(x_{j}, t_{n}\right)$. Then the truncation error of difference schemes (63)-(65) is as follows:

$$
\begin{aligned}
& r_{j}^{n}=\left(v_{j}^{n}\right)_{\widehat{t}}+\left(v_{j}^{n}\right)_{x x \overline{x x} \hat{t}} \\
&+\left(\bar{v}_{j}^{n}\right)_{\widehat{x}}+\left(\bar{v}_{j}^{n}\right)_{x \bar{x} \widehat{x}}-\left(\bar{v}_{j}^{n}\right)_{x x \overline{x x} \hat{x}}+\alpha \psi\left(v_{j}^{n}, \bar{v}_{j}^{n}\right), \\
&(j=1,2, \ldots, J-1, n=1,2, \ldots, N-1), \\
& v_{j}^{0}=u_{0}\left(x_{j}\right), \quad(j=0,1,2, \ldots, J), \\
& v^{n} \in Z_{h}^{0}, \quad\left(v_{0}^{n}\right)_{\widehat{x}}=\left(v_{J}^{n}\right)_{\widehat{x}}=0, \\
&\left(v_{0}^{n}\right)_{x \bar{x}}=\left(v_{J}^{n}\right)_{x \bar{x}}=0, \quad(n=0,1,2, \ldots, N) .
\end{aligned}
$$

By Taylor expansion, we know that $r_{j}^{n}=O\left(\tau^{2}+h^{2}\right)$ as $h, \tau \rightarrow$ 0 .

Theorem 14. Suppose that $u_{0} \in H_{0}^{2}\left[x_{L}, x_{R}\right]$. Then the solution of difference schemes (63)-(65) satisfies $\left\|u^{n}\right\| \leq C,\left\|u_{x}^{n}\right\| \leq C$, $\left\|u_{x x}^{n}\right\| \leq C$; thus $\left\|u^{n}\right\|_{\infty} \leq C,\left\|u_{x}^{n}\right\|_{\infty} \leq C(n=1,2, \ldots, N)$.

Proof. From (67), we know that

$$
\left\|u^{n}\right\| \leq C, \quad\left\|u_{x x}^{n}\right\| \leq C .
$$

By (38), one can get

$$
\left\|u_{x}^{n}\right\| \leq C
$$

Then, from Lemma 6, we get $\left\|u^{n}\right\|_{\infty} \leq C,\left\|u_{x}^{n}\right\|_{\infty} \leq C$.

Remark 15. Theorem 14 implies that the solution of difference schemes (63)-(65) is unconditionally stable in the sense of norm $\|\cdot\|_{\infty}$.

Theorem 16. Suppose that $u_{0} \in H_{0}^{2}\left[x_{L}, x_{R}\right]$. Then the solution of difference schemes (63)-(65) $u^{n}$ converges to the solution of (2)-(4) in the sense of norm $\|\cdot\|_{\infty}$, and the convergent order is $O\left(\tau^{2}+h^{2}\right)$.

Proof. Subtracting (63)-(65) from (83)-(85) and letting $e_{j}^{n}=$ $v_{j}^{n}-u_{j}^{n}$, we have

$$
\begin{gathered}
r_{j}^{n}=\left(e_{j}^{n}\right)_{\hat{t}}+\left(e_{j}^{n}\right)_{x x \overline{x x} \hat{t}}+\left(\bar{e}_{j}^{n}\right)_{\widehat{x}}+\left(\bar{e}_{j}^{n}\right)_{x \bar{x} \widehat{x}} \\
-\left(\bar{e}_{j}^{n}\right)_{x x \overline{x x} \widehat{x}}+\alpha \psi\left(v_{j}^{n}, \bar{v}_{j}^{n}\right)-\alpha \psi\left(u_{j}^{n}, \bar{u}_{j}^{n}\right), \\
(j=1,2, \ldots, J-1, n=1,2, \ldots, N-1), \\
e_{j}^{0}=u_{0}\left(x_{j}\right), \quad(j=0,1,2, \ldots, J), \\
e^{n} \in Z_{h}^{0}, \quad\left(e_{0}^{n}\right)_{\widehat{x}}=\left(e_{J}^{n}\right)_{\widehat{x}}=0, \\
\left(e_{0}^{n}\right)_{x \bar{x}}=\left(e_{J}^{n}\right)_{x \bar{x}}=0, \quad(n=0,1,2, \ldots, N) .
\end{gathered}
$$

Taking the inner product of the two sides of (88) with $2 \bar{e}^{n}$, and using boundary condition (90) and Lemma 1, we obtain

$$
\begin{aligned}
\left\langle r^{n}, 2 \bar{e}^{n}\right\rangle= & \left\|e^{n}\right\|_{\hat{t}}^{2}+\left\|e_{x x}^{n}\right\|_{\hat{t}}^{2}+\left\langle\bar{e}_{\widehat{x}}^{n}, 2 \bar{e}^{n}\right\rangle+\left\langle\bar{e}_{x \bar{x} \hat{x}}^{n}, \bar{e}^{n}\right\rangle \\
& -\left\langle\bar{e}_{x x \bar{x} \bar{x} \hat{x}}^{n}, 2 \bar{e}^{n}\right\rangle+\alpha\left\langle\psi\left(v^{n}, \bar{v}^{n}\right)-\psi\left(u^{n}, \bar{u}^{n}\right), 2 \bar{e}^{n}\right\rangle .
\end{aligned}
$$

Similar to (71)-(73), we have

$$
\begin{gathered}
\left\langle\bar{e}_{\widehat{x}}^{n}, 2 \bar{e}^{n}\right\rangle=0, \\
\left\langle\bar{e}_{x \bar{x} \hat{x}}^{n}, 2 \bar{e}^{n}\right\rangle=0, \\
\left\langle\bar{e}_{x x \overline{x x} \hat{x}}^{n}, 2 \bar{e}^{n}\right\rangle=0 .
\end{gathered}
$$

Then (91) is changed into

$$
\begin{aligned}
& \left(\left\|e^{n+1}\right\|^{2}-\left\|e^{n-1}\right\|^{2}\right)+\left(\left\|e_{x x}^{n+1}\right\|^{2}-\left\|e_{x x}^{n-1}\right\|^{2}\right) \\
& \quad=2 \tau\left\langle r^{n}, 2 \bar{e}^{n}\right\rangle-2 \alpha \tau\left\langle\psi\left(v^{n}, \bar{v}^{n}\right)-\psi\left(u^{n}, \bar{u}^{n}\right), 2 \bar{e}^{n}\right\rangle .
\end{aligned}
$$

By Lemma 5 and Theorem 14, it is shown that

$$
\left|\bar{v}_{j}^{n}\right| \leq C, \quad\left|u_{j}^{n}\right| \leq C, \quad\left|\left(u_{j}^{n}\right)_{\widehat{x}}\right| \leq C, \quad(j=0,1,2, \ldots, J) .
$$

Using Cauchy-Schwarz inequality, we have

$$
\begin{aligned}
-\alpha & \left\langle\psi\left(v^{n}, \bar{v}^{n}\right)-\psi\left(u^{n}, \bar{u}^{n}\right), 2 \bar{e}^{n}\right\rangle \\
= & \frac{-2 h}{3} \alpha \sum_{j=1}^{J-1}\left[v_{j}^{n}\left(\bar{v}_{j}^{n}\right)_{\widehat{x}}-u_{j}^{n}\left(\bar{u}_{j}^{n}\right)_{\widehat{x}}\right] \bar{e}_{j}^{n} \\
& -\frac{2 h}{3} \alpha \sum_{j=1}^{J-1}\left[\left(v_{j}^{n} \bar{v}_{j}^{n}\right)_{\widehat{x}}-\left(u_{j}^{n} \bar{u}_{j}^{n}\right)_{\widehat{x}}\right] \bar{e}_{j}^{n} \\
= & \frac{-2 h}{3} \alpha \sum_{j=1}^{J-1}\left[v_{j}^{n}\left(\bar{v}_{j}^{n}\right)_{\widehat{x}}-u_{j}^{n}\left(\bar{u}_{j}^{n}\right)_{\widehat{x}}\right] \bar{e}_{j}^{n} \\
& +\frac{2 h}{3} \alpha \sum_{j=1}^{J-1}\left(v_{j}^{n} \bar{v}_{j}^{n}-u_{j}^{n} \bar{u}_{j}^{n}\right)\left(\bar{e}_{j}^{n}\right)_{\widehat{x}} \\
= & \frac{-2 h}{3} \alpha \sum_{j=1}^{J-1}\left[e_{j}^{n}\left(\bar{v}_{j}^{n}\right)_{\widehat{x}}+u_{j}^{n}\left(\bar{e}_{j}^{n}\right)_{\widehat{x}}\right] \bar{e}_{j}^{n} \\
& +\frac{2 h}{3} \alpha \sum_{j=1}^{J-1}\left(e_{j}^{n} \bar{v}_{j}^{n}+u_{j}^{n} \bar{e}_{j}^{n}\right)\left(e_{j}^{n}\right)_{\widehat{x}} \\
\leq & C\left(\left\|e^{n}\right\|^{2}+\left\|\bar{e}^{n}\right\|^{2}+\left\|\bar{e}_{x}^{n}\right\|^{2}\right) \\
\leq & C\left(\left\|e^{n+1}\right\|^{2}+\left\|e^{n}\right\|^{2}+\left\|e^{n-1}\right\|^{2}+\left\|e_{x}^{n+1}\right\|^{2}+\left\|e_{x}^{n-1}\right\|^{2}\right) .
\end{aligned}
$$

On the other hand,

$$
\begin{aligned}
\left\langle r^{n}, 2 \bar{e}^{n}\right\rangle & =\left\langle r^{n}, e^{n+1}+e^{n-1}\right\rangle \\
& \leq\left\|r^{n}\right\|^{2}+\frac{1}{2}\left(\left\|e^{n+1}\right\|^{2}+\left\|e^{n-1}\right\|^{2}\right) .
\end{aligned}
$$


Substituting (95)-(96) into (93), we could obtain

$$
\begin{gathered}
\left(\left\|e^{n+1}\right\|^{2}-\left\|e^{n-1}\right\|^{2}\right)+\left(\left\|e_{x x}^{n+1}\right\|^{2}-\left\|e_{x x}^{n-1}\right\|^{2}\right) \\
\leq C \tau\left(\left\|e^{n+1}\right\|^{2}+\left\|e^{n}\right\|^{2}+\left\|e^{n-1}\right\|^{2}+\left\|e_{x}^{n+1}\right\|^{2}\right. \\
\left.+\left\|e_{x}^{n}\right\|^{2}+\left\|e_{x}^{n-1}\right\|^{2}\right)+2 \tau\left\|r^{n}\right\|^{2} .
\end{gathered}
$$

Using the deduce process which is similar to (38), we have

$$
\begin{aligned}
\left\|e_{x}^{n+1}\right\|^{2} & \leq \frac{1}{2}\left(\left\|e^{n+1}\right\|^{2}+\left\|e_{x x}^{n+1}\right\|^{2}\right), \\
\left\|e_{x}^{n}\right\|^{2} & \leq \frac{1}{2}\left(\left\|e^{n}\right\|^{2}+\left\|e_{x x}^{n}\right\|^{2}\right), \\
\left\|e_{x}^{n-1}\right\|^{2} & \leq \frac{1}{2}\left(\left\|e^{n-1}\right\|^{2}+\left\|e_{x x}^{n-1}\right\|^{2}\right) .
\end{aligned}
$$

Then (97) is changed into

$$
\begin{gathered}
\left(\left\|e^{n+1}\right\|^{2}-\left\|e^{n-1}\right\|^{2}\right)+\left(\left\|e_{x x}^{n+1}\right\|^{2}-\left\|e_{x x}^{n-1}\right\|^{2}\right) \\
\leq C \tau\left[\left\|e^{n+1}\right\|^{2}+\left\|e^{n}\right\|^{2}+\left\|e^{n-1}\right\|^{2}+\left\|e_{x x}^{n+1}\right\|^{2}\right. \\
\left.+\left\|e_{x x}^{n}\right\|^{2}+\left\|e_{x x}^{n-1}\right\|^{2}\right]+2 \tau\left\|r^{n}\right\|^{2} .
\end{gathered}
$$

Let $B^{n}=\left\|e^{n+1}\right\|^{2}+\left\|e_{x x}^{n+1}\right\|^{2}+\left\|e^{n}\right\|^{2}+\left\|e_{x x}^{n}\right\|^{2},(99)$ can be rewritten as follows:

$$
B^{n}-B^{n-1} \leq C \tau\left(B^{n}+B^{n-1}\right)+2 \tau\left\|r^{n}\right\|^{2}
$$

that is,

$$
(1-C \tau)\left(B^{n}-B^{n-1}\right) \leq 2 C \tau B^{n-1}+2 \tau\left\|r^{n}\right\|^{2} .
$$

By choosing $\tau$ that is small enough such that $1-C \tau>0$, then

$$
B^{n}-B^{n-1} \leq C \tau B^{n-1}+C \tau\left\|r^{n}\right\|^{2} .
$$

Summing up (102) from 1 to $n$, we obtain

$$
B^{n} \leq B^{0}+C \tau \sum_{l=1}^{n}\left\|r^{l}\right\|^{2}+C \tau \sum_{l=0}^{n-1} B^{l}
$$

Notice that

$$
\tau \sum_{l=0}^{n-1}\left\|r^{l}\right\|^{2} \leq n \tau \max _{0 \leq l \leq n-1}\left\|r^{l}\right\|^{2} \leq T \cdot O\left(\tau^{2}+h^{2}\right)^{2} .
$$

Firstly by selecting other two-level scheme with second-order convergence (such as the scheme (9)-(11)) to get $u^{1}$, we have

$$
B^{0}=O\left(\tau^{2}+h^{2}\right)^{2}
$$

Therefore

$$
B^{n} \leq O\left(\tau^{2}+h^{2}\right)^{2}+C \tau \sum_{l=0}^{n-1} B^{l}
$$

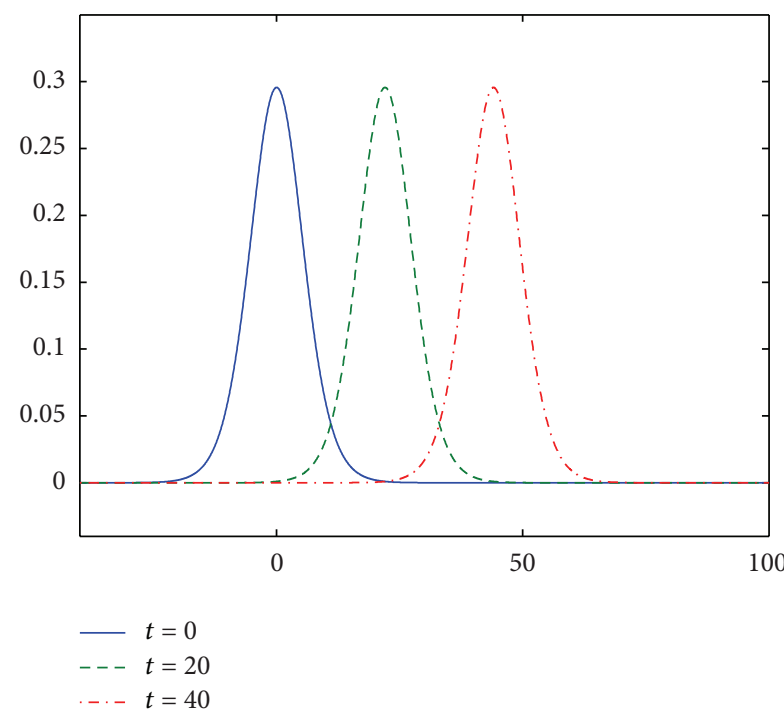

FIGURE 1: When $\alpha=1$ and $\tau=h=0.05$, a numerical simulation figure on $u(x, t)$ of Scheme I at various times.

Using Lemma 7,we obtain

$$
B^{n} \leq O\left(\tau^{2}+h^{2}\right)^{2}
$$

that is,

$$
\left\|e^{n}\right\| \leq O\left(\tau^{2}+h^{2}\right), \quad\left\|e_{x x}^{n}\right\| \leq O\left(\tau^{2}+h^{2}\right) .
$$

Combining with (98), we get

$$
\left\|e_{x}^{n}\right\| \leq O\left(\tau^{2}+h^{2}\right) .
$$

Then by Lemma 6, it is shown that

$$
\left\|e^{n}\right\|_{\infty} \leq O\left(\tau^{2}+h^{2}\right) .
$$

\section{Numerical Simulations}

In this section, let $\alpha=1, x_{L}=-40, x_{R}=100, T=40$, and

$$
u_{0}(x)=\left(-\frac{35}{12}+\frac{35}{156} \sqrt{205}\right) \operatorname{sech}^{4}\left(\frac{1}{12} \sqrt{-13+\sqrt{205} x}\right) .
$$

We denote the nonlinear Crank-Nicolson conservative difference schemes (9)-(11) as Scheme I and the linear threelevel conservative difference schemes (63)-(65) as Scheme II. For some different values of $\tau$ and $h$, we list errors of Scheme I and Scheme II at several different time in Tables 1 and 2, respectively. We verify the two-order accuracy of the difference scheme in Tables 3 and 4 by using the method of $[26,27]$. The numerical simulation of two conservative quantities (6) and (7) is listed in Tables 5 and 6.

Finally, a numerical simulation figure comparison of $u(x, t)$ at various time steps with $\tau=h=0.05$ is as follows. (see Figures 1 and 2). 
TABLE 1: The errors of Scheme I at various time steps with various $h$ and $\tau$.

\begin{tabular}{|c|c|c|c|c|c|c|}
\hline & \multicolumn{2}{|c|}{$\tau=h=0.1$} & \multicolumn{2}{|c|}{$\tau=h=0.05$} & \multicolumn{2}{|c|}{$\tau=h=0.025$} \\
\hline & $\left\|e^{n}\right\|$ & $\left\|e^{n}\right\|_{\infty}$ & $\left\|e^{n}\right\|$ & $\left\|e^{n}\right\|_{\infty}$ & $\left\|e^{n}\right\|$ & $\left\|e^{n}\right\|_{\infty}$ \\
\hline$t=10$ & $2.159730 e-4$ & $7.520810 e-5$ & $5.401591 e-5$ & $1.880684 e-5$ & $1.354230 e-5$ & $4.702168 e-6$ \\
\hline$t=20$ & $4.160331 e-4$ & $1.421127 e-4$ & $1.040460 e-4$ & $3.554032 e-5$ & $2.603381 e-5$ & $8.886828 e-6$ \\
\hline$t=30$ & $5.977491 e-4$ & $1.996835 e-4$ & $1.494989 e-4$ & $4.994370 e-5$ & $3.739276 e-5$ & $1.248752 e-5$ \\
\hline$t=40$ & $7.633356 e-4$ & $2.502663 e-4$ & $1.909241 e-4$ & $6.260150 e-5$ & $4.774838 e-5$ & $1.565301 e-5$ \\
\hline
\end{tabular}

TABLE 2: The errors of Scheme II at various time steps with various $h$ and $\tau$.

\begin{tabular}{lcccccc}
\hline & \multicolumn{2}{c}{$\tau=h=0.1$} & \multicolumn{2}{c}{$\tau=h=0.05$} & \multicolumn{2}{c}{$\tau=h=0.025$} \\
& $\left\|e^{n}\right\|$ & $\left\|e^{n}\right\|_{\infty}$ & $\left\|e^{n}\right\|$ & $\left\|e^{n}\right\|_{\infty}$ & $\left\|e^{n}\right\|$ & $\left\|e^{n}\right\|_{\infty}$ \\
\hline$t=10$ & $4.302763 e-4$ & $1.477136 e-4$ & $1.076811 e-4$ & $3.697042 e-5$ & $2.693880 e-5$ & $9.259288 e-6$ \\
$t=20$ & $8.320777 e-4$ & $2.820308 e-4$ & $2.082744 e-4$ & $7.060528 e-5$ & $5.207086 e-5$ & $1.767754 e-5$ \\
$t=30$ & $1.199482 e-3$ & $3.985869 e-4$ & $3.002761 e-4$ & $9.979291 e-5$ & $7.504636 e-5$ & $2.497799 e-5$ \\
$t=40$ & $1.535826 e-3$ & $5.014481 e-4$ & $3.845124 e-4$ & $1.255601 e-4$ & $9.606941 e-5$ & $3.141830 e-5$ \\
\hline
\end{tabular}

TABle 3: The verification of the second convergence of Scheme I.

\begin{tabular}{|c|c|c|c|c|c|c|}
\hline & \multicolumn{3}{|c|}{$\left\|e^{n}(h, \tau)\right\| /\left\|e^{2 n}(h / 2, \tau / 2)\right\|$} & \multicolumn{3}{|c|}{$\left\|e^{n}(h, \tau)\right\|_{\infty} /\left\|e^{2 n}(h / 2, \tau / 2)\right\|_{\infty}$} \\
\hline & $\tau=h=0.1$ & $\tau=h=0.05$ & $\tau=h=0.025$ & $\tau=h=0.1$ & $\tau=h=0.05$ & $\tau=h=0.025$ \\
\hline$t=10$ & - & 3.998318 & 3.988313 & - & 3.998969 & 3.999234 \\
\hline$t=20$ & - & 3.998548 & 3.996548 & - & 3.998630 & 3.999538 \\
\hline$t=30$ & - & 3.998359 & 3.998072 & - & 3.998171 & 3.999489 \\
\hline$t=40$ & - & 3.998109 & 3.998457 & - & 3.997769 & 3.999324 \\
\hline
\end{tabular}

TABLE 4: The verification of the second convergence of Scheme II.

\begin{tabular}{cccccc}
\hline & & $\left\|e^{n}(h, \tau)\right\| /\left\|e^{2 n}(h / 2, \tau / 2)\right\|$ & \multicolumn{2}{c}{$\left\|e^{n}(h, \tau)\right\|_{\infty} /\left\|e^{2 n}(h / 2, \tau / 2)\right\|_{\infty}$} \\
& $\tau=h=0.1$ & $\tau=h=0.05$ & $\tau=h=0.025$ & $\tau=h=0.1$ & $\tau=h=0.05$ \\
\hline$t=10$ & - & 3.995835 & 3.997182 & - & 3.995455 \\
$t=20$ & - & 3.995103 & 3.999826 & - & 3.994473 \\
$t=30$ & - & 3.994599 & 4.001208 & - & 3.994140 \\
$t=40$ & - & 3.994217 & 4.002444 & - & 3.993009 \\
\hline
\end{tabular}

TABLE 5: When $\alpha=1$, numerical simulations on the conservation invariant $Q^{n}$.

\begin{tabular}{lcccccc}
\hline & \multicolumn{2}{c}{$\tau=h=0.1$} & \multicolumn{2}{c}{$\tau=h=0.05$} & \multicolumn{2}{c}{$\tau=h=0.025$} \\
& Scheme I & Scheme II & Scheme I & Scheme II & Scheme I & Scheme II \\
\hline$t=0$ & 4.120893241330 & 4.120918187462 & 4.120893214999 & 4.120899452450 & 4.120893201770 & 4.120894761190 \\
$t=10$ & 4.120892312503 & 4.120918835632 & 4.120892200166 & 4.120898816174 & 4.120892129735 & 4.120894227434 \\
$t=20$ & 4.120893031520 & 4.120921893266 & 4.120892362862 & 4.120899447408 & 4.120892167548 & 4.120894662524 \\
$t=30$ & 4.120893107802 & 4.120925120669 & 4.120892370454 & 4.120900136940 & 4.120892149140 & 4.120895106600 \\
$t=40$ & 4.120892997828 & 4.120924996792 & 4.120892342248 & 4.120900073781 & 4.120892155018 & 4.120895399389 \\
\hline
\end{tabular}

TABLE 6: When $\alpha=1$, numerical simulations on the conservation invariant $E^{n}$.

\begin{tabular}{lccccrr}
\hline & \multicolumn{2}{c}{$\tau=h=0.1$} & \multicolumn{2}{c}{$\tau=h=0.05$} & \multicolumn{2}{c}{$\tau=h=0.025$} \\
& Scheme I & Scheme II & Scheme I & Scheme II & \multicolumn{2}{c}{ Scheme I } \\
\hline$t=0$ & 0.836201094485 & 0.836201094485 & 0.836201181724 & 0.836201181724 & 0.836201203535 & 0.836201203535 \\
$t=10$ & 0.836201095943 & 0.836201096547 & 0.836201194492 & 0.836201202559 & 0.836201310312 & 0.836201469358 \\
$t=20$ & 0.836201095943 & 0.836201096662 & 0.836201194483 & 0.836201206568 & 0.836201308626 & 0.836201594118 \\
$t=30$ & 0.836201095952 & 0.836201096780 & 0.836201194484 & 0.836201210387 & 0.836201309421 & 0.836201718674 \\
$t=40$ & 0.836201095955 & 0.836201096906 & 0.836201194369 & 0.836201214174 & 0.836201309604 & 0.836201843455 \\
\hline
\end{tabular}




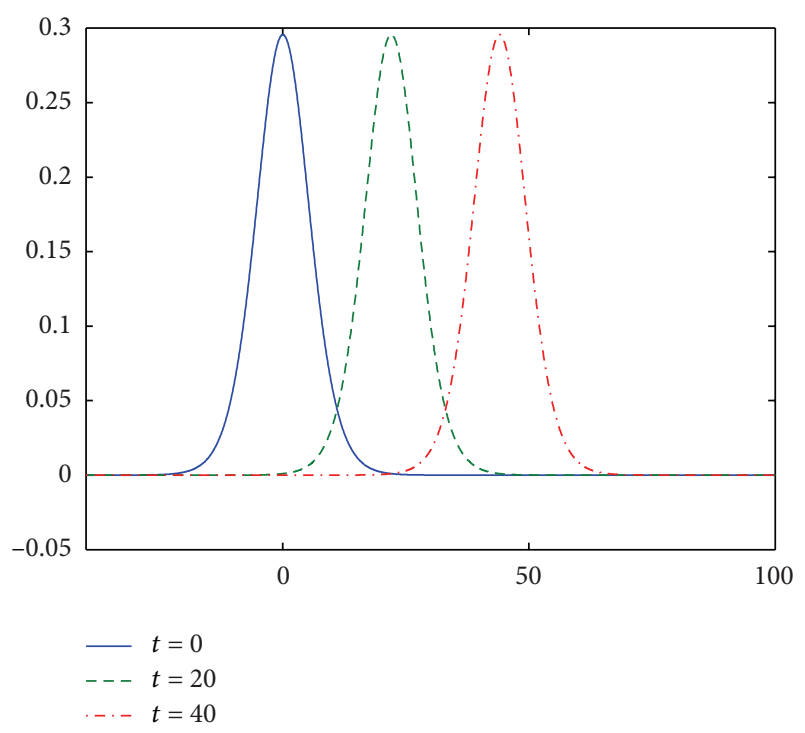

Figure 2: When $\alpha=1$ and $\tau=h=0.05$, a numerical simulation figure on $u(x, t)$ of Scheme II at various times.

Numerical simulations show that the finite difference Schemes I and II in this paper are efficient. The calculation results of Scheme I are slightly better than Scheme II. But iterative numerical calculation is not required, Scheme II can save computing time.

\section{Conflict of Interests}

The authors declare that there is no conflict of interests regarding the publication of this paper.

\section{Acknowledgments}

This work is supported by the National Natural Science Foundation of China (11171239), Open Fund of Key Laboratory of Mountain Hazards and Earth Surface Processes, CAS and Scientific Research Found of Sichuan Provincial Education Department (no. 11ZB009).

\section{References}

[1] P. Rosenau, "A quasi-continuous description of a nonlinear transmission line," Physica Scripta, vol. 34, pp. 827-829, 1986.

[2] P. Rosenau, "Dynamics of dense discrete systems," Progress of Theoretical Physics, vol. 79, pp. 1028-1042, 1988.

[3] M. A. Park, "On the Rosenau equation," Matemática Aplicada e Computacional, vol. 9, no. 2, pp. 145-152, 1990.

[4] S. K. Chung and S. N. Ha, "Finite element Galerkin solutions for the Rosenau equation," Applicable Analysis, vol. 54, no. 1-2, pp. 39-56, 1994.

[5] K. Omrani, F. Abidi, T. Achouri, and N. Khiari, "A new conservative finite difference scheme for the Rosenau equation," Applied Mathematics and Computation, vol. 201, no. 1-2, pp. 3543, 2008.
[6] S. K. Chung, "Finite difference approximate solutions for the Rosenau equation," Applicable Analysis, vol. 69, no. 1-2, pp. 149$156,1998$.

[7] S. K. Chung and A. K. Pani, "Numerical methods for the Rosenau equation," Applicable Analysis, vol. 77, no. 3-4, pp. 351369, 2001.

[8] S. A. V. Manickam, A. K. Pani, and S. K. Chung, "A second-order splitting combined with orthogonal cubic spline collocation method for the Rosenau equation," Numerical Methods for Partial Differential Equations, vol. 14, no. 6, pp. 695-716, 1998.

[9] Y. D. Kim and H. Y. Lee, "The convergence of finite element Galerkin solution for the Roseneau equation," The Korean Journal of Computational \& Applied Mathematics, vol. 5, no. 1, pp. 171-180, 1998.

[10] J.-M. Zuo, "Solitons and periodic solutions for the Rosenau$\mathrm{KdV}$ and Rosenau-Kawahara equations," Applied Mathematics and Computation, vol. 215, no. 2, pp. 835-840, 2009.

[11] M. Labidi and A. Biswas, "Application of He's principles to Rosenau-Kawahara equation," Mathematics in Engineering, Science and Aerospace MESA, vol. 2, no. 2, pp. 183-197, 2011.

[12] A. Biswas, H. Triki, and M. Labidi, "Bright and dark solitons of the Rosenau-Kawahara equation with power law nonlinearity," Physics of Wave Phenomena, vol. 19, no. 1, pp. 24-29, 2011.

[13] T. Wang, L. Zhang, and F. Chen, "Conservative schemes for the symmetric regularized long wave equations," Applied Mathematics and Computation, vol. 190, no. 2, pp. 1063-1080, 2007.

[14] S. Li and L. Vu-Quoc, "Finite difference calculus invariant structure of a class of algorithms for the nonlinear Klein-Gordon equation," SIAM Journal on Numerical Analysis, vol. 32, no. 6, pp. 1839-1875, 1995.

[15] Q. Chang, E. Jia, and W. Sun, "Difference schemes for solving the generalized nonlinear Schrödinger equation," Journal of Computational Physics, vol. 148, no. 2, pp. 397-415, 1999.

[16] T.-C. Wang and L.-M. Zhang, "Analysis of some new conservative schemes for nonlinear Schrödinger equation with wave operator," Applied Mathematics and Computation, vol. 182, no. 2, pp. 1780-1794, 2006.

[17] T. Wang, B. Guo, and L. Zhang, "New conservative difference schemes for a coupled nonlinear Schrödinger system," Applied Mathematics and Computation, vol. 217, no. 4, pp. 1604-1619, 2010.

[18] L. Zhang, "A finite difference scheme for generalized regularized long-wave equation," Applied Mathematics and Computation, vol. 168, no. 2, pp. 962-972, 2005.

[19] Z. Fei and L. Vázquez, "Two energy conserving numerical schemes for the sine-Gordon equation," Applied Mathematics and Computation, vol. 45, no. 1, pp. 17-30, 1991.

[20] Y. S. Wong, Q. Chang, and L. Gong, "An initial-boundary value problem of a nonlinear Klein-Gordon equation," Applied Mathematics and Computation, vol. 84, no. 1, pp. 77-93, 1997.

[21] Q. S. Chang, B. L. Guo, and H. Jiang, "Finite difference method for generalized Zakharov equations," Mathematics of Computation, vol. 64, no. 210, pp. 537-553, 1995.

[22] J. Hu and K. Zheng, "Two conservative difference schemes for the generalized Rosenau equation," Boundary Value Problems, vol. 2010, Article ID 543503, 18 pages, 2010.

[23] X. Pan and L. Zhang, "On the convergence of a conservative numerical scheme for the usual Rosenau-RLW equation," Applied Mathematical Modelling, vol. 36, no. 8, pp. 3371-3378, 2012. 
[24] X. Pan and L. Zhang, "Numerical simulation for general Rosenau-RLW equation: an average linearized conservative scheme," Mathematical Problems in Engineering, vol. 2012, Article ID 517818, 15 pages, 2012.

[25] F. E. Browder, "Existence and uniqueness theorems for solutions of nonlinear boundary value problems," in Proceedings of Symposia in Applied Mathematics, vol. 17, pp. 24-49, 1965.

[26] T. Wang and B. Guo, "A robust semi-explicit difference scheme for the Kuramoto-Tsuzuki equation," Journal of Computational and Applied Mathematics, vol. 233, no. 4, pp. 878-888, 2009.

[27] J. $\mathrm{Hu}, \mathrm{B}$. $\mathrm{Hu}$, and $\mathrm{Y}$. $\mathrm{Xu}$, " $\mathrm{C}-\mathrm{N}$ difference schemes for dissipative symmetric regularized long wave equations with damping term," Mathematical Problems in Engineering, vol. 2011, Article ID 651642, 16 pages, 2011. 


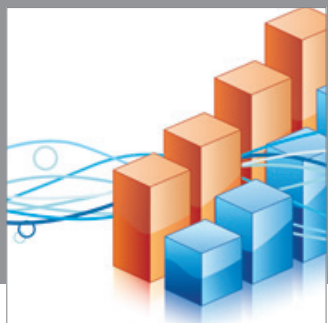

Advances in

Operations Research

mansans

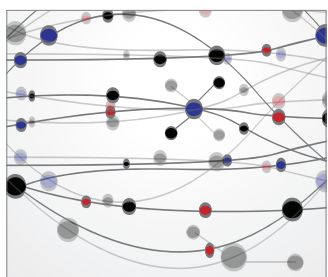

The Scientific World Journal
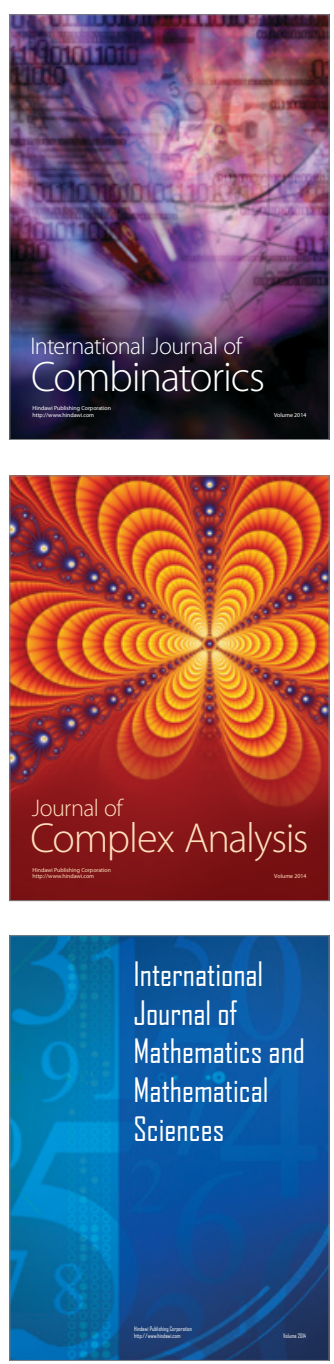
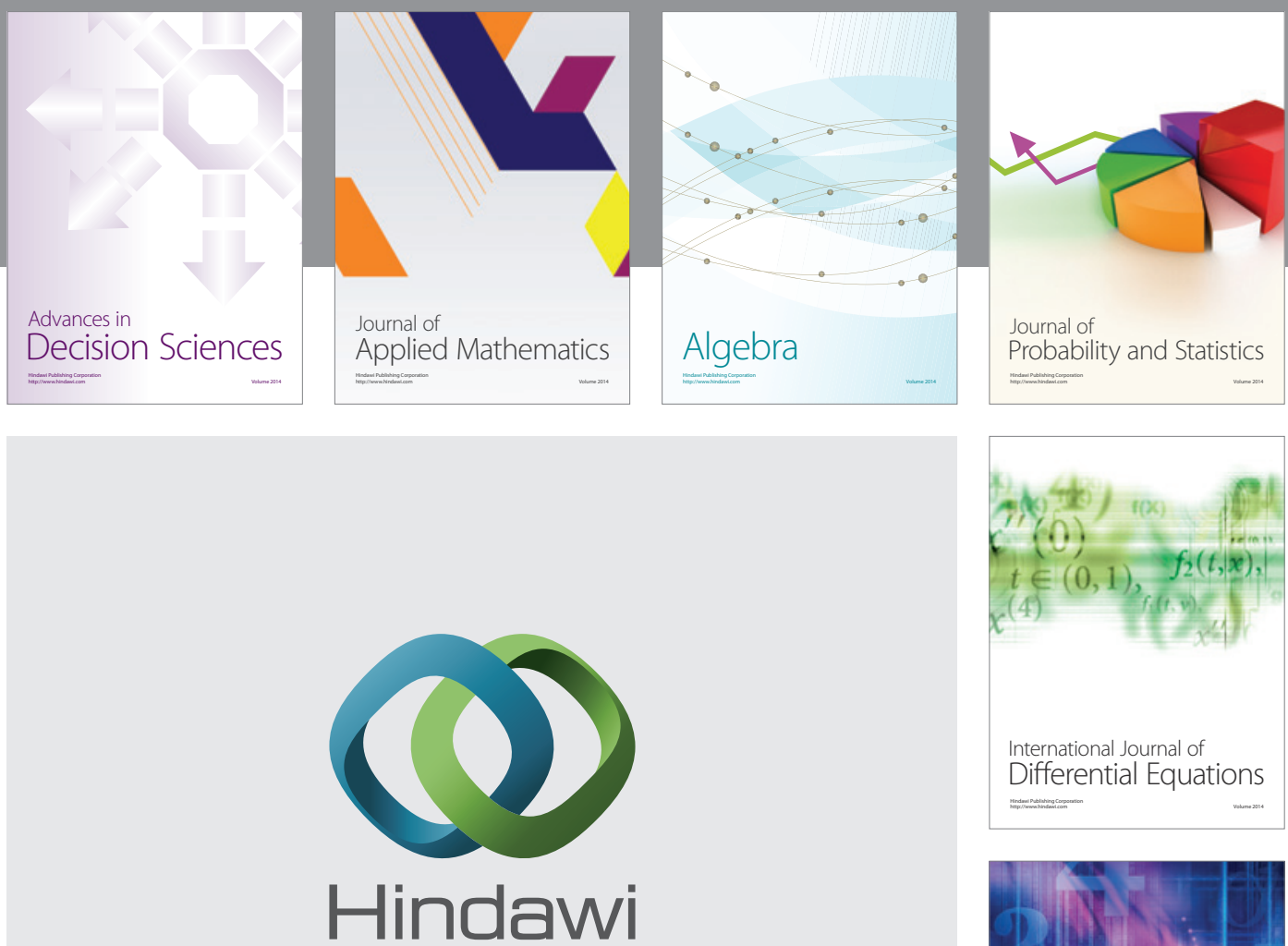

Submit your manuscripts at http://www.hindawi.com
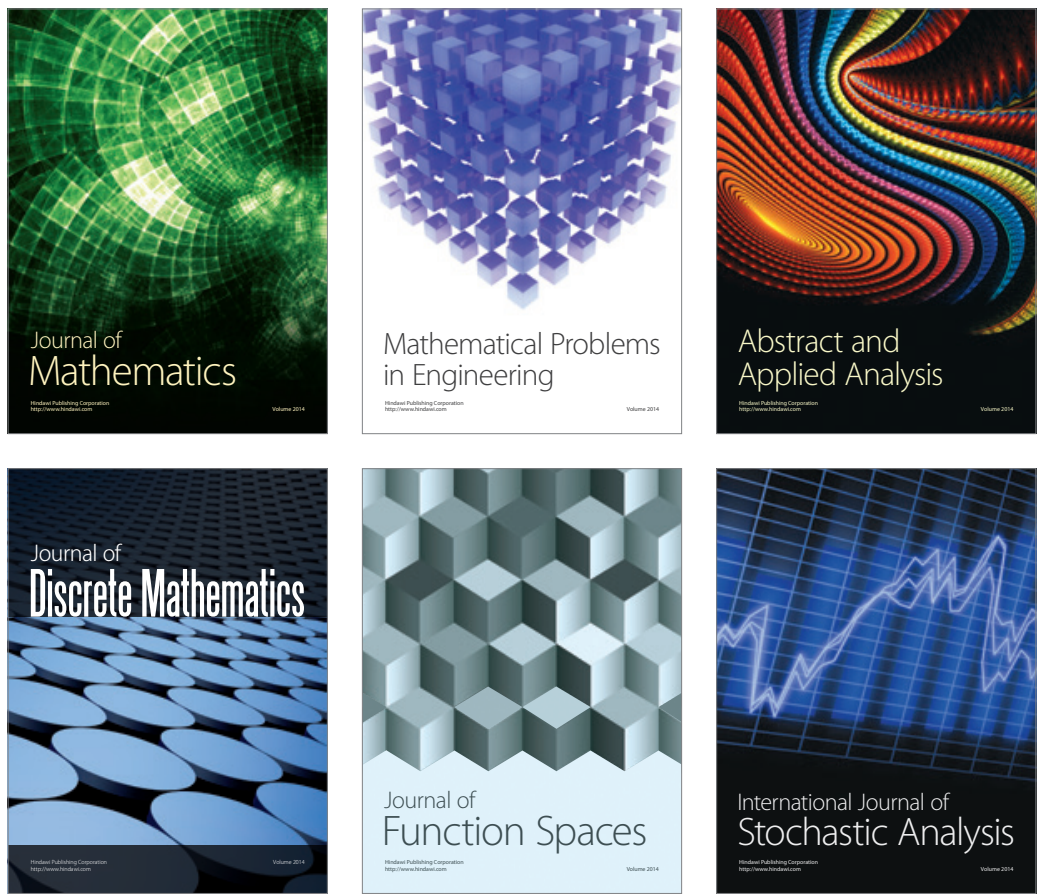

Journal of

Function Spaces

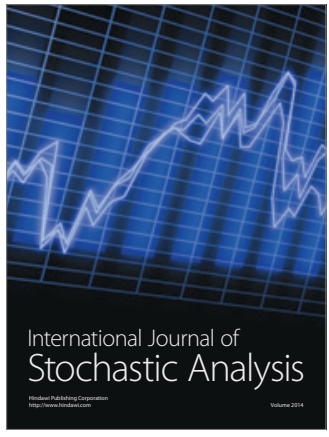

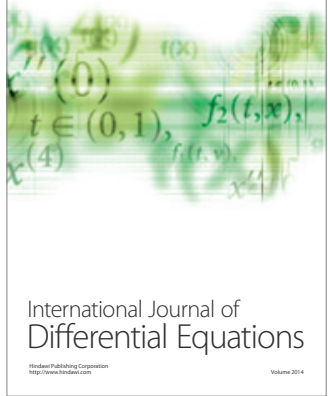
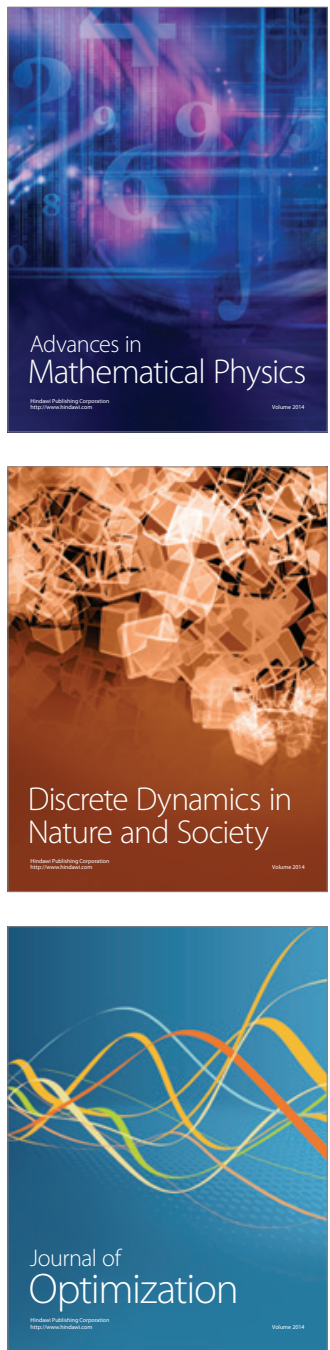